less IL-1ß, and the expression of anti-inflammatory genes, such as IL-10, was upregulated. Furthermore, treatment with BTK inhibitor increased the rate of phagocytosis by antiinflammatory M2 macrophages in vitro.

Conclusions Our findings show that BTK inhibition hinders M1 macrophage differentiation and skews monocytes towards an anti-inflammatory M2 phenotype, while enhancing apoptotic cell uptake by M2 cells. Therefore, BTK inhibition could have additional benefits in the treatment of autoimmune diseases such as SLE, by targeting both B cells and myeloid cells simultaneously.

Funding Source(s): This study was sponsored by EMD Serono Inc. (a business of Merck KGaA, Darmstadt, Germany)

\title{
217 CLINICAL AND SEROLOGICAL PROFILE OF A SERIES OF RHUPUS PATIENTS
}

${ }^{1}$ Beatriz Frade Sosa* ${ }^{2}$ Vera Ortiz-Santamaría, ${ }^{3}$ Vicente Torrente-Segarra, ${ }^{4}$ Ivan Castellvi, ${ }^{4}$ Berta Magallares, ${ }^{5}$ Delia Reina, ${ }^{6}$ Sonia Minguez, ${ }^{7}$ Meritxell Sallés, ${ }^{8}$ Sergi Ordoñez, ${ }^{9}$ Elena Riera, ${ }^{10}$ Maria Garcia Manrique, ${ }^{11}$ Jose A Gómez-Puerta. ${ }^{1}$ Rheumatology Department, Hospital Clinic, Barcelona; ${ }^{2}$ Hospital General de Granollers; ${ }^{3}$ Hospital Comarcal de l'Alt Penedès; ${ }^{4}$ Hospital Santa Creu i Sant Pau; ${ }^{5}$ Hospital Sant Joan Despi Moisès Broggi; ${ }^{6}$ AlTHAIA, Xarxa Assistencial Universitària de Manresa; ${ }^{7}$ ALTHAIA, Xarxa Assistencial Universitària de Manresa, Spain; ${ }^{8}$ Hospital Arnau de Vilanova, Lleida; ${ }^{9}$ Hospital Mutua de Terrassa; ${ }^{10}$ Hospital Parc Tauli, Sabadell; ${ }^{11}$ Rheumatology Department, Hospital Clinic, Barcelona, Spain

\subsection{6/lupus-2019-Ism.217}

Background Concomitant presence of two autoimmune diseases, such as rheumatoid arthritis (RA) and systemic lupus erythematosus (SLE) is known as Rhupus. Despite,

Abstract 217 Table 1 General characteristics of Rhupus and SLE patients

\begin{tabular}{|c|c|c|c|}
\hline & Rhupus ( $n=19$ ) & SLE $(n=38)$ & $P$ value \\
\hline Gender (Female), \% & $17(89.5)$ & $36(94.7)$ & 0.59 \\
\hline Mean age, years $\pm S D$ & $56.9 \pm 12.8$ & $45.9 \pm 12.3$ & 0.03 \\
\hline Disease duration, years $\pm S D$ & $13.9 \pm 7.0$ & $10.5 \pm 9.7$ & 0.24 \\
\hline Race (Caucasian), \% & $14(73)$ & $27(71.1)$ & 0.89 \\
\hline \multicolumn{4}{|l|}{ Clinical characteristics } \\
\hline Oral ulcers, $\%$ & $2(10.5)$ & $16(42.1)$ & $<0.01$ \\
\hline Articular involvement, \% & $19(100)$ & $36(94.7)$ & $<0.01$ \\
\hline - Arthritis, \% & $19(100)$ & $29(76.3)$ & 0.02 \\
\hline - Erosive disease, \% & $11(57.9)$ & $1(2.6)$ & $<0.01$ \\
\hline - Tenosynovitis, \% & $10(52.6)$ & $19(26.3)$ & 0.05 \\
\hline Leukopenia, \% & $3(15.8)$ & $21(55.3)$ & $<0.01$ \\
\hline Renal involvement, \% & $1(5.3)$ & $10(26.3)$ & 0.07 \\
\hline Mean SLEDAI * & $1.2 \pm 1.6$ & $3.3 \pm 3.4$ & 0.03 \\
\hline \multicolumn{4}{|l|}{ Immunological features } \\
\hline Mean RF levels, IU \pm SD & $184.6 \pm 199.3$ & $47.6 \pm 114.5$ & $<0.01$ \\
\hline Mean anti-CCP titers, IU \pm SD & $622.3 \pm 908.5$ & $5.1 \pm 5.2$ & $<0.01$ \\
\hline Positive anti-Ro antibodies, \% & $15(18.9)$ & $17(48.6)$ & 0.03 \\
\hline \multicolumn{4}{|l|}{ Treatment (ever) } \\
\hline Prednisolone, \% & $19(100)$ & $28(75.7)$ & 0.02 \\
\hline Methotrexate, \% & $17(89.5)$ & $13(36.1)$ & $<0.01$ \\
\hline Rituximab, \% & $8(44.4)$ & $5(14.7)$ & 0.04 \\
\hline
\end{tabular}

poliautoimmunity is not uncommon described in patients with systemic autoimmune diseases, only a small series of patients have been described so far with Rhupus. Our purpose was to analyze clinical and serological characteristics of patients with Rhupus and compare them with a cohort of patients with SLE.

Methods In this cross-sectional study, we included cases of Rhupus (RA-ACR/EULAR 2010 plus SLE-ACR 1987 criteria) from different Rheumatology Departments at Catalonia, Spain. In addition, we included patients with diagnosis of SLE in a 2:1 ratio matched by sex and race. All information was recorded following an established protocol.

Results A total of 57 patients were included, 19 cases with Rhupus and 38 cases of SLE alone as controls. 93\% of patients were female, Caucasian represented $71.4 \%$, Mestizo $17.9 \%$ and $5.4 \%$ were Asian. Mean age was $48.6 \pm 13.5$ years and mean disease duration was $11.48 \pm 9.1$ years. Main clinical characteristics were cutaneous involvement (75.0\%), hematological (66.0\%), serositis (19.3\%), renal disease $(17.9 \%)$ and secondary Sjögren syndrome (28\%) among others. Clinical and serological characteristics according groups are shown in table 1 .

Conclusions We found some clinical and serological differences among patients with Rhupus and SLE alone. As expected, articular domains and titers of RF and ACPAs were higher in Rhupus and they are more commonly treated with methotrexate and rituximab. By other hand, leukopenia, oral ulcers, anti-Ro antibodies and higher SLEDAI score were more common among SLE patients. Whether Rhupus patients represent a different condition requires further analysis in bigger cohorts.

\section{THERAPEUTIC TRAJECTORIES FOLLOWING HIGH DISEASE ACTIVITY STATE IN SLE}

${ }^{1}$ Christopher McMaster, ${ }^{1}$ Hieu Nim, ${ }^{1}$ Rachel Koelmeyer, ${ }^{2}$ Albert Frauman, ${ }^{3}$ Eric Morand, ${ }^{1}$ Alberta Y Hoi*. ${ }^{1}$ Monash University; ${ }^{2}$ Melbourne University; ${ }^{3}$ Monash University, Melbourne, Australia

\subsection{6/lupus-2019-Ism.218}

Background Systemic lupus erythematosus (SLE) is a heterogenous autoimmune disease. Treatment trajectories following high disease activity state (HDAS), as defined by SLEDAI score 10 , have not been well described.

Methods Longitudinal trajectories of patients from the Australian Lupus Registry were studied. HDAS periods were defined as the time from which HDAS begins, until the patient fulfils criteria for Low Lupus Disease Activity (LLDAS), or up to 365 days. Treatment escalation is defined as either an addition of hydroxychloroquine (HCQ), prednisolone (PNL) and immunosuppressant (IS), or any change in IS drug. De-escalation is either dose reduction or cessation of HCQ or IS without meeting treatment escalation criteria. Treatment trajectories were examined as the rolling sum (over time) of escalations and de-escalations and were clustered using k-means clustering methods. Different clustering partitions were tested. The $\mathrm{R}$ package $\mathrm{kml}$ was used for cluster determination and quality criterion calculations. The differences in time to resolution of HDAS between clusters were tested using likelihood ratio test. 